INPLASY

PROTOCOL

To cite: Wang et al.

Effectiveness of Different Nutritional Methods for Poststroke dysphagia: An Updated Systematic Review and Metaanalysis. Inplasy protocol

202220014. doi:

10.37766/inplasy2022.2.0014

Received: 06 February 2022

Published: 06 February 2022

Corresponding author:

Heping Li

peacewar2005@163.com

Author Affiliation:

The First Affiliated Hospital of

Zhengzhou University.

Support: Non-profit Central

Research.

Review Stage at time of this submission: Data analysis.

Conflicts of interest:

None declared.

\section{Effectiveness of Different Nutritional Methods for Post-stroke dysphagia: An Updated Systematic Review and Meta-analysis}

\author{
Wang, S1; Zhang, Q2; Zeng, X³; Li, HX4.
}

Review question / Objective: Post-stroke Dysphagia seriously affects people's quality of life, among which malnutrition and pulmonary infection are the most common complications of post-stroke Dysphagia. At this point, it is very important to choose the right auxiliary feeding mode. The purpose of this meta-analysis was to study the prognosis of patients with dysphagia after stroke who chose different nutritional methods.

Information sources: We searched the Cochrane Library, PubMed, Medline, China National Knowledge Infrastructure (CNKI), Wanfang, and the VIP database.

INPLASY registration number: This protocol was registered with the International Platform of Registered Systematic Review and Meta-Analysis Protocols (INPLASY) on 06 February 2022 and was last updated on 06 February 2022 (registration number INPLASY202220014).

\section{INTRODUCTION}

Review question / Objective: Post-stroke Dysphagia seriously affects people's quality of life, among which malnutrition and pulmonary infection are the most common complications of post-stroke Dysphagia. At this point, it is very important to choose the right auxiliary feeding mode. The purpose of this meta-analysis was to study the prognosis of patients with dysphagia after stroke who chose different nutritional methods.

Condition being studied: Effectiveness of Different Nutritional Methods for Post- 
stroke dysphagia. This study has 2 associate senior title or above staff, 1 doctoral graduate and 1 master student, all of whom have high abilities in literature retrieval, reading and content extraction.

\section{METHODS}

Participant or population: Patients with dysphagia after stroke (1115).

Intervention: A mode of supplementary nutrition.

Comparator: Different assisted feeding modes from the intervention group.

Study designs to be included: RCTs.

Eligibility criteria: According to the imaging data (brain CT or MRI) or the relevant diagnostic criteria mentioned in the article.

Information sources: We searched the Cochrane Library, PubMed, Medline, China National Knowledge Infrastructure (CNKI), Wanfang, and the VIP database.

Main outcome(s): Nutritional indexes (Hb,Alb levels), pulmonary infection rate, recovery of swallowing function.

Quality assessment / Risk of bias analysis: The Newcastle-Ottawa-Scale (NOS) score was used to evaluate the literature quality. The risk of bias was assessed using the Cochrane instrument.

Strategy of data synthesis: All statistical analyses were performed using RevMan software 5.4 .We considered $12<30 \%$ low statistical heterogeneity, $\geq 30 \%$ to $<50 \%$ moderate heterogeneity, $\geq 50 \%$ to $<75 \%$ substantial heterogeneity, and $\geq 75 \%$ considerable heterogeneity.

Subgroup analysis: Subgroup analysis has not been considered.

Sensitivity analysis: Sensitivity analysis was performed by RevMan. A sensitivity analysis was also conducted, in which 1 study at a time was removed and the other analyzed estimate whether the results could have been affected markedly by a single study. This analysis confirmed the stability of our results.

Country(ies) involved: China.

Keywords: Stroke, dysphagia, nutritional mode, intermittent oral to esophageal tube feeding, nasal feeding tube, percutaneous endoscopic gastrostomy.

Contributions of each author:

Author 1 - Shaowei Wang.

Email: wangshaowei1026@163.com

Author 2 - Qiongshuai Zhang.

Author 3 - Xi Zeng.

Author 4 - Heping Li.

Email: peacewar2005@163.com 